\title{
EFFICACY OF HYDROXYCHLOROQUINE IN THE PREVENTION OF THROMBOEMBOLIC EVENTS: A SYSTEMATIC REVIEW AND
}

\section{META-ANALYSIS}

Fernando Luiz Barros Edington ${ }^{1,2, \star}$, Rayssa Valandro Garcia ${ }^{1}$, Luis Fernando Simões dos Santos ${ }^{1}$, Daniel Fraga de Rezende ${ }^{1}$, Sandra Rocha Gadelha Mello ${ }^{1}$, Mittermayer Barreto Santiago ${ }^{1,2}$

1.Universidade Estadual de Santa Cruz, Ilhéus (BA), Brazil; 2.Escola Bahiana de Medicina e Saúde Pública, Salvador (BA), Brazil.

*Corresponding author: fernandoedington@hotmail.com

\section{BACKGROUND}

Hydroxychloroquine was introduced in the early 1940s for the treatment of malaria and, since then, its use has been expanded to other diseases as rheumatoid arthritis, lupus and antiphospholipid syndrome. In 1941, Carter A.E. et al. postulated that the erythrocyte "desludging" effect attributed to hydroxychloroquine could also decrease the propensity of platelets to adhere and form clots, reducing the incidence of deep venous thrombosis and pulmonary embolism. Considering its low cost, general good safety profile, the data already published and the unavailability of a systematic review on this theme, we decided to systematically review the efficacy of hydroxychloroquine in the prevention of thromboembolic events.

\section{METHODS}

We systematically reviewed randomized controlled trials (RCTs), using MEDLINE and EMBASE databases from inception to the present, reporting thromboembolic events in hydroxychloroquine users compared to nonusers. Four authors independently screened all the records obtained through our search strategy and later revised the selected full-text articles for eligibility, according to our inclusion criteria. The same four authors independently extracted relevant data through a customized data collection form while two other authors assessed the quality of the included RCTs using the Cochrane risk-of-bias tool (version 2.0). All the disagreements were resolved by discussion among the authors. We then calculated the relative risk (RR) and its respective standard error of developing thromboembolic events in antimalarials users and nonusers for each individual study and pooled the results using a random effectmodel meta-analysis. We assessed Heterogeneity using the Tau2 and I2, and publication bias using funnel plotting and Egger's regression. The protocol for this systematic review is registered at the PROSPERO database (CRD42021247902).

\section{RESULTS}

Thirteen RCTs met our eligibility criteria and were included in our analysis (2,663 patients). We found that hydroxychloroquine reduced the risk of thromboembolic events by $47 \%(R R=0.53$, IC $95 \%=0.34-0.83)$ (Figure 1$)$ with a medium heterogeneity $(12=75 \%$ and $\mathrm{T} 2=0.37)$. We did not find a significant asymmetry in the inspection of the funnel plot (Figure 2$)$, which was later confirmed through an Egger's regression ( $p=0.1025)$.

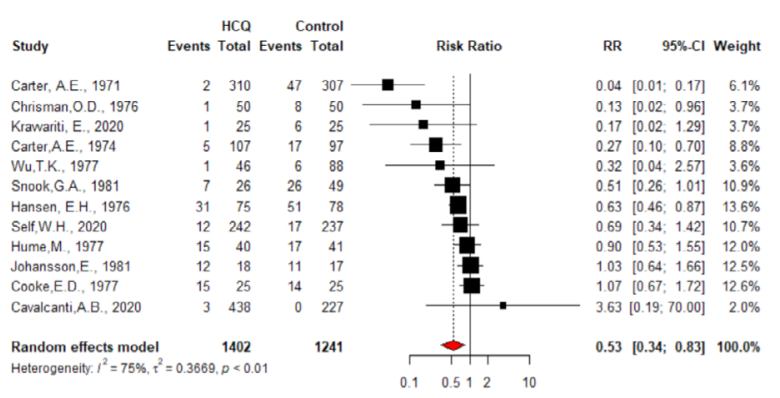

Figure 1. Relative risk of thromboembolic events in hydroxychloroquine users compared to nonusers.

Realização: 


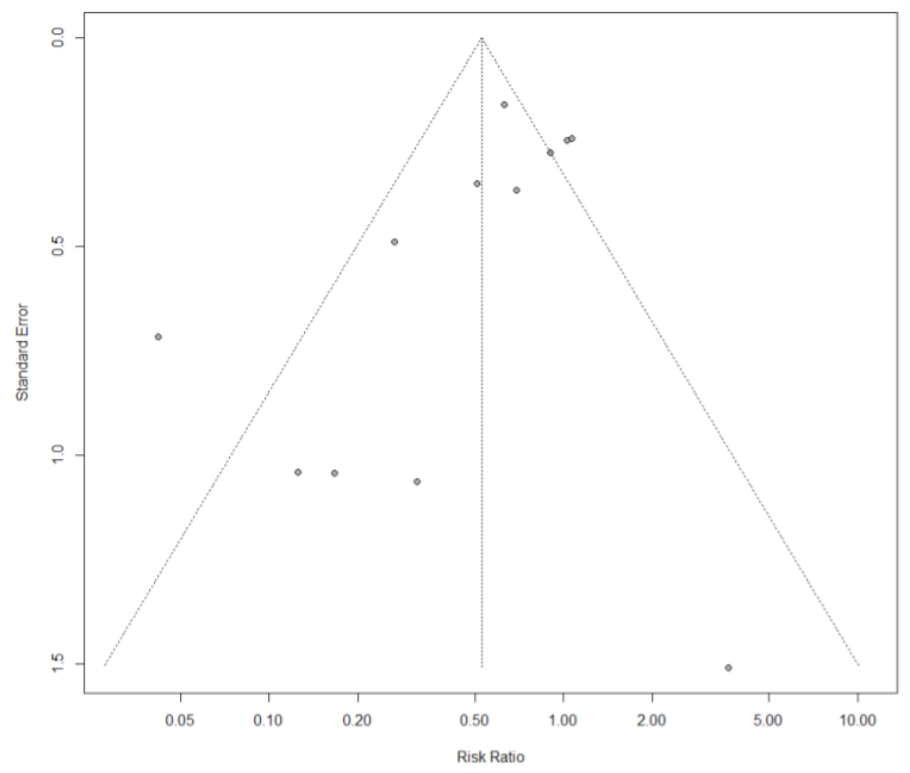

Figure 2. Funnel-plot ( $p$-value $=0.1025)$.

\section{CONCLUSION}

Our data reinforces the idea that hydroxychloroquine reduces the risk of thromboembolic events.

\section{KEYWORDS}

Hydroxychloroquine, Anticoagulants, Systematic-review, Meta-analysis. 